\title{
ON AN ERGODIC PROPERTY OF A CERTAIN CLASS OF MARKOV PROCESSES
}

\section{GOPINATH KALLIANPUR}

1. Introduction. We consider a spatially and temporally homogeneous separable Markov process $x_{s}$ with the transition probability function

$$
P(s, \xi ; A)=\operatorname{Prob}\left(x_{s+s_{0}} \in A / x_{s_{0}}=\xi\right),
$$

which will be assumed to satisfy the following conditions:

$$
P(s, \xi ; A)=\int_{A} p(s, x-\xi) d x .
$$

There exists a positive function $k(t)$ tending to infinity with $t$ such that

$$
k(t) p(t, u) \leqq C,
$$

$$
\begin{aligned}
\lim _{t \rightarrow \infty} \frac{k(t+a)}{k(t)} & =1 \\
\sum_{n=1}^{\infty} \frac{1}{k(n)} & =\infty,
\end{aligned}
$$

for any constant $a$,

(1.5) For fixed $u$,

$$
\lim _{t \rightarrow \infty} k(t) p(t, u)=1
$$

and

(1.6) For fixed $u, p(t, u)$ is a continuous function of $t$ for $t$ different from zero.

The above conditions on the transition function are suggested by applications to the Brownian motion and, in the discrete case, to sums of independent random variables belonging to the domain of attraction of symmetric stable laws. There are Markov processes besides the Brownian motion for which conditions (1.1) to (1.6) are fulfilled, e.g. the Cauchy process with

$$
p(t, u)=t \pi^{-1}\left(t^{2}+u^{2}\right)^{-1} .
$$

The main object of this paper is to prove an ergodic or "equidistribution" property of the Brownian motion process, a weak form of

Received by the editors March 4, 1954. 
which was proved in [1]. The problem for discrete Markov chains has been studied by Harris and Robbins [2]. The method adopted here is not a generalization of their method for the continuous case but relies on the specific properties of the density function assumed in (1.1) to (1.6). The reason for this approach lies firstly in the fact that we are primarily concerned here with the Brownian motion. Secondly, in the case of sums of independent random variables belonging to the domain of attraction of symmetric stable laws, we are able with our method to obtain a necessary and sufficient condition for equidistribution in terms of the norming constants. More specific comments will be made in $\$ 5$.

From (1.1), (1.4), and (1.5) it is easily verified that for fixed $\xi$ and for fixed linear bounded Borel set $A$,

$$
\lim _{s \rightarrow \infty} P(s, \xi ; A)=0,
$$

while

$$
\int_{-\infty}^{\infty} P(s, \xi ; A) d \xi=l(A),
$$

$l$ being Lebesgue measure. Thus the Markov processes we consider admit an infinite invariant measure (see [2]).

2. Definition of the measure $m$. Let $\Omega$ be the space of real functions $x_{8}(-\infty<s<\infty), F$ the field of finite unions of cylinders of the form

$$
W=\left[x_{s_{0}} \in A_{0}, \cdots, x_{s_{k}} \in A_{k}\right],
$$

and $B(F)$ the Borel extension of $F$. Here and in the sequel the sets which appear within the braces in (2.1) are linear bounded Borel sets and the suffixes under the letter $x$ are arranged in increasing order of magnitude. For sets of the form (2.1) let $Q\left(W / x_{s_{0}}=\xi\right)$ denote the conditional probability of $W$ given $x_{s_{0}}=\xi$. Then

$$
m(W)=\int_{-\infty}^{\infty} Q\left(W / x_{s_{0}}=\xi\right) d \xi
$$

defines $m$ as a measure over $F$. Just as in the discrete case [2], since $\Omega$ itself is the denumerable union of sets in $F$ of finite $m$-measure, $m$ can be extended uniquely to $B(F)$ by the Kolmogorov extension theorem. If $W$ is a cylinder, (2.2) defines $m(W)$ in a unique manner in the sense that the right side of (2.2) gives the same value if $s_{0}$ is replaced by $s_{0}^{\prime}$, where $0<s_{0}^{\prime} \leqq s_{0}$. We now define a group $T_{t}(-\infty<t$ $<\infty)$ of coordinate shift transformations, i.e.,

$$
T_{t} x_{8}=x_{8+t} .
$$


We shall denote the corresponding set transformation by the same letter (see [3, p. 453]). It is easy to show that for $W \in B(F)$,

$$
m\left(T_{\imath} W\right)=m(W) .
$$

For later purposes we need the following

LEMMA. If $V$ and $W$ are Borel cylinders of finite measure, then $m\left(V \cdot T_{t} W\right)$ is a continuous function of $t$ for $t \neq 0$.

If $V, W \in F$, the proof follows on a successive application of the Lebesgue dominated convergence theorem. Its extension to $V, W$ $\in B(F)$ is straightforward. For instance if $V \in F$ and $W \in B(F)$, let $W_{\delta} \in F$ be such that

$$
m\left(W-W_{\delta}\right)+m\left(W_{\delta}-W\right)<\delta .
$$

Then from the relation

$$
\begin{aligned}
\left|m\left(V \cdot T_{t} W\right)-m\left(V \cdot T_{\tau} W\right)\right| \leqq & \left|m\left(V \cdot T_{t} W\right)-m\left(V \cdot T_{t} W_{\delta}\right)\right| \\
& +\left|m\left(V \cdot T_{t} W_{\delta}\right)-m\left(V \cdot T_{\tau} W_{\delta}\right)\right| \\
& +\left|m\left(V \cdot T_{\tau} W\right)-m\left(V \cdot T_{\tau} W_{\delta}\right)\right|
\end{aligned}
$$

we have

$$
\lim _{t \rightarrow \tau} m\left(V \cdot T_{\imath} W\right)=m\left(V \cdot T_{\tau} W\right) \quad(\tau \neq 0) .
$$

We do not need the continuity of $m\left(V \cdot T_{t} W\right)$ at $t=0$ for our purpose and indeed it may not be obtained in many cases.

In the next section we prove two theorems which enable us to apply the continuous form of Hopf's ergodic theorem [4, pp. 53-54].

3. Absence of the dissipative part and metric transitivity of $T_{t}$.

Theorem 1. The dissipative part of $\Omega$ has zero m-measure.

Proof. Let $V, W \in F$ be of positive finite measure. Then

$$
\lim _{t \rightarrow \infty} k(t) m\left(V \cdot T_{t} W\right)=m(V) m(W) .
$$

Before proving (3.1) we make two remarks.

(i) (3.1) holds trivially if the right side is zero.

(ii) If

$$
m\left(V \cdot T_{\imath} W\right)=0 \quad \text { for all sufficiently large } t_{\mathrm{v}}
$$

then with $W$ as in (2.1) and

$$
V=\left[x_{u_{0}} \in B_{0}, \cdots, x_{u_{r}} \in B_{r}\right],
$$


we have for all large $t$

$$
\begin{aligned}
k(t) m\left(V \cdot T_{t} W\right)= & k(t) m\left(W \cdot T_{-t} V\right) \\
= & \frac{k(t)}{k\left(t+u_{0}-s_{k}\right)} \int_{A_{0}} d \xi \int_{A_{1}} P\left(s_{1}-s_{0}, \xi ; d \xi_{1}\right) \cdots \\
& \cdot \int_{A_{k}} P\left(s_{k}-s_{k-1}, \xi_{k-1} ; d \xi_{k}\right) \\
& \cdot \int_{B_{0}} k\left(t+u_{0}-s_{k}\right) p\left(t+u_{0}-s_{k}, \eta-\xi_{k}\right) \\
& \cdot h(\eta) d \eta,
\end{aligned}
$$

where $h(\eta)=\int_{B_{1}} P\left(u_{1}-u_{0}, \eta ; d \eta_{1}\right) \cdots \int_{B_{k}} P\left(u_{k}-u_{k-1}, \eta_{k-1} ; d \eta_{k}\right)$.

On account of (3.2) the right side of (3.3) is zero, which implies that either

$$
m(V)=0
$$

or that

$$
\begin{array}{r}
\int_{B_{0}} k\left(t+u_{0}-s_{k}\right) p\left(t+u_{0}-s_{k}, \eta-\xi_{k}\right) h(\eta) d \eta=0 \\
\text { for a.e. } \xi_{k} \text { in } A_{k} \text { (Lebesgue measure). }
\end{array}
$$

But from (1.5) for every fixed $x$,

$$
k\left(t+u_{0}-s_{k}\right) p\left(t+u_{0}-s_{k}, x\right)>0 \quad \text { for all sufficiently large } t .
$$

Hence either $B_{0}$ is of zero measure, or

$$
h(\eta)=0 \quad \text { for a.e. } \eta \text { in } B_{0} .
$$

Either of these conclusions implies $m(W)=0$. Hence under (3.2) we have

$$
m(V) m(W)=0 .
$$

In order to prove (3.1) when $V$ and $W$ are $F$-sets of positive finite measure it is enough to make $t \rightarrow \infty$ on the right side of (3.3) and apply (1.4) and (1.5).

Let $V, W_{j}(j=1,2, \cdots) \in F$ be of positive finite measure and let $M=\sum_{j=1}^{\infty} W_{j}$. From (3.1)

$$
\begin{aligned}
\lim _{t \rightarrow \infty} k(t) m\left(\sum_{1}^{N} W_{j} \cdot T_{-t} V\right) & =m\left(\sum_{1}^{N} W_{j}\right) m(V) \\
& \geqq m\left(W_{1}\right) m(V)>0 .
\end{aligned}
$$


Hence for every $N$ and all sufficiently large $t$,

$$
k(t) m\left(\sum_{1}^{N} W_{j} \cdot T_{-t} V\right) \geqq K>0 .
$$

Making $N \rightarrow \infty$ we obtain

$$
k(t) m\left(V \cdot T_{t} M\right) \geqq K>0 \quad \text { for all large } t .
$$

(3.5) remains true for every $M \in B(F)$ of finite measure, for then there exists a set $M_{0}$ which is a denumerable union of finite $F$-sets such that $M_{0} \supset M$ and $m\left(M_{0}-M\right)=0$. From (3.5) and (1.3), taking $t=n$ integral, we find that if

$$
m\left(V \cdot T_{n} M\right)>0
$$

for all but a finite number of values of $n$, then

$$
\sum_{n=1}^{\infty} m\left(V \cdot T_{n} M\right)
$$

diverges. If $\Omega_{1}$ is the dissipative part of $\Omega$ [4, pp. 46-49] we can write $\Omega_{1}=\sum_{r} T_{r} M, M$ being a wandering set and $r$ taking all rational values. $M$ may be taken to be of finite measure.

Consider now a fixed set $V \in F$ of positive, finite measure. Then

$$
m\left(V \cdot \sum_{n=-\infty}^{\infty} T_{n} M\right)=\sum_{n=-\infty}^{\infty} m\left(V \cdot T_{n} M\right)<\infty
$$

since $m(V)<\infty$. But in view of (3.6) this can be the case only if

$$
m\left(V \cdot T_{n} M\right)=0
$$

for an infinity of values $n$.

Now because of $\sigma$-finiteness

$$
\Omega=\sum_{i=-\infty}^{\infty} V_{i}, \quad V_{i} \in F \quad\left(0<m\left(V_{i}\right)<\infty\right) .
$$

Since the $V_{i}$ 's are denumerable it is clear from (3.7) that

$$
m\left(V_{i} \cdot T_{n} M\right)=0
$$

for an infinite sequence of values of $n$ which is the same for all $i$. Denoting this sequence by $n_{1}, n_{2}, \cdots$, we have

$$
m\left(T_{n_{j}} M\right) \leqq \sum_{i=-\infty}^{\infty} m\left(V_{i} \cdot T_{n_{j}} M\right)=0
$$

from (3.8). Hence 


$$
m(M)=m\left(T_{n j} M\right)=0,
$$

which implies that $\Omega_{1}$ has zero measure.

TheOREM 2. The group $T_{t}$ is metrically transitive.

We divide the proof into two cases.

Case 1. Let $W$ be an invariant set of finite measure. Then there exists a set $M_{0}=\sum_{j=1}^{\infty} A_{j}\left(A_{j} \in F\right)$ such that $M_{0} \supset W$ and $m\left(M_{0}-W\right)$ $=0$. For every rational $r, T_{r} W=W \subset T_{r} M_{0}$ and $m\left(T_{r} M_{0}-W\right)=0$. Setting

$$
M=\sum_{r} T_{r} M_{0}=\sum_{r} \sum_{i} T_{r} A_{j} \quad\left(T_{r} A_{j} \in F\right)
$$

we have

$$
m\left[\left(M-T_{t} M\right)+\left(T_{t} M-M\right)\right]=0
$$

for rational $t$ since in that case $T_{t} M=M$. From the continuity of $M\left(V \cdot T_{t} W\right)(t \neq 0)$ proved in the lemma it follows that $(3.10)$ holds for all $t$. Hence $T_{t} M=M$. Furthermore

$$
W \subset M \text { and } m(M-W)=0
$$

since $M-W=\sum_{r}\left(T_{r} M_{0}-W\right)$, a denumerable union of sets of zero measure. By an argument similar to the one used in Theorem 1,

$$
\limsup _{t \rightarrow \infty} k(t) m(V \cdot M) \leqq C<\infty
$$

( $C$ being a constant) for every $V \in F$ of finite positive measure. Taking $V=T_{r} A_{j},(3.12)$ can hold only if $m\left(T_{r} A_{j}\right)=m\left(T_{r} A_{j} \cdot M\right)=0$ for every $j=1,2, \cdots$ and for every rational $r$. Hence $m(M)=0$ and from (3.11), $m(W)=0$.

Case 2. Let the invariant set $W$ be of infinite measure. Since $\Omega$ is the denumerable union of sets of finite measure, we may write

$$
\Omega-W=\sum_{j} A_{j} \quad \text { and } \quad W=\sum_{j} B_{j},
$$

where the sets $A_{j}$ and $B_{j}$ are of finite measure.

For each $j$ there exists an $M_{j}=\sum_{k=1}^{\infty} M_{j k}\left(M_{j k} \in F\right)$ with $M_{j} \supset A_{j}$ and $m\left(M_{j}-A_{j}\right)=0$.

Assuming as we may that $B_{1}$ has positive measure, let $C_{N}$ be the ascending sequence of $F$-sets converging to $C \supset B_{1}$ where $m\left(C-B_{1}\right)$ $=0$. Then

$$
\begin{aligned}
m\left(M_{j k} \cdot T_{t} C_{N}\right) & \leqq m\left(M_{j k} \cdot T_{t} C\right)=m\left(M_{j k} \cdot T_{t} B_{1}\right) \\
& \leqq m\left(M_{j k} \cdot T_{t} W\right)=m\left(M_{j k} \cdot W\right) \leqq m\left(A_{j} \cdot W\right)=0
\end{aligned}
$$


from (3.13). (3.2), (3.4), and (3.14) then imply

$$
m\left(M_{j k}\right) m\left(C_{N}\right)=0,
$$

from which we obtain $m\left(M_{j k}\right)=0$ for $k=1,2, \cdots$. Hence $m\left(M_{j}\right)$ $=m\left(A_{j}\right)=0$ for every $j$ thus proving that $m(\Omega-W)=0$. This completes the proof of Theorem 2.

Let $f(u)$ and $g(u)$ be summable functions of a real variable $u$ with $\int_{-\infty}^{\infty} g(u) d u \neq 0$. Then Theorems 1 and 2 and Hopf's theorem imply

Theorem 3. For almost all $x_{0}$ (Lebesgue measure)

$$
\lim _{T \rightarrow \infty} \frac{\int_{0}^{T} f\left(x_{t}\right) d t}{\int_{0}^{T} g\left(x_{t}\right) d t}=\frac{\int_{-\infty}^{\infty} f(u) d u}{\int_{-\infty}^{\infty} g(u) d u}
$$

with probability one.

4. Application to the Brownian motion. Let $a$ be an arbitrary real number and $y_{t}(t \geqq 0)$ the sample function of the separable Brownian motion process. In this case conditions (1.1) to (1.6) are satisfied with

$$
p(t, u)=(2 \pi t)^{-1 / 2} e^{-\mu^{2} / 2 t} \text {, and } k(t)=(2 \pi t)^{1 / 2} .
$$

Setting

$$
x_{t}=a+y_{t}
$$

Theorem 3 gives

TheOREM 4. ${ }^{1}$ If $y_{t}$ is the Brownian motion process, then for a.e. a (Lebesgue measure)

$$
\lim _{T \rightarrow \infty} \frac{\int_{0}^{T} f\left(a+y_{t}\right) d t}{\int_{0}^{T} g\left(a+y_{t}\right) d t}=\frac{\int_{-\infty}^{\infty} f(u) d u}{\int_{-\infty}^{\infty} g(u) d u}
$$

with probability one.

5. Application to sums of independent random variables. Let $y_{j}(j=1,2, \cdots)$ be independent random variables with the common distribution function $F(y)$. Under the assumption that the partial sums $y_{1}+\cdots+y_{n}$ are interval recurrent it is proved in [2] that

1 Added in proof: The author understands that C. Derman, using a different method, has also obtained Theorem 4 in which the result holds with $a=0$. 
(5.1) Prob $\left[\lim _{n \rightarrow \infty} \frac{\sum_{j=1}^{n} f\left(a+y_{1}+\cdots+y_{j}\right)}{\sum_{j=1}^{n} g\left(a+y_{1}+\cdots+y_{j}\right)}=\frac{\int f(u) d u}{\int g(u) d u}\right]=1$

for a.e. $a$ (Lebesgue measure), and furthermore that (5.1) holds for every $a$ if $F(y)$ has an absolutely continuous component. However, it may not be easy to verify the hypothesis of interval recurrence in particular cases. Let us assume that the probability density $F^{\prime}(y)$ exists and satisfies the following conditions (see [5]):

$$
F^{\prime}(y) \in L^{p}(-\infty, \infty) \quad \text { for some } p>1 \text {; }
$$

(5.3) There exist positive constants $B_{n}$ such that

$$
\lim _{n \rightarrow \infty} \operatorname{Prob}\left(\frac{y_{1}+\cdots+y_{n}}{B_{n}} \leqq y\right)=V_{\alpha}(y),
$$

$V_{\alpha}(y)$ being the symmetric stable law with exponent $\alpha(0<\alpha \leqq 2)$. (If $\alpha=2$ we assume $E y^{2}<\infty$.)

If $\alpha>1, E|y|$ is finite and a criterion of Chung and Fuchs [6] ensures interval recurrence provided $E y=0$, while if $B_{n}=$ Constant $\cdot n^{1 / \alpha}$ $(0<\alpha<1)$ the partial sums are not interval recurrent [5]. With regard to the other possibilities (for instance when $\alpha=1$ ) we do not know whether the sufficient conditions given in [6] always apply. A similar situation arises when the $y$ 's are integer-valued. Hence it seems desirable to obtain a condition involving the constants $B_{n}$ under which (5.1) is true.

Assuming (5.2) and (5.3) to hold, the density $p_{n}(y)$ of $y_{1}+\cdots+y_{n}$ satisfies (Lemma 6.1 of [5])

(5.4) $B_{n} p_{n}(y) \leqq C$ independent of $n$ and $y$, and

$$
\lim _{n \rightarrow \infty} B_{n}\left\{\Gamma\left(\alpha^{-1}\right)\right\}^{-1} p_{n}(y)=1
$$

for every $y$.

Thus the discrete analogue of (1.4) and (1.5) is fulfilled. On account of (5.3) the norming constants $B_{n}$ satisfy $[7$, p. 155]

$$
B_{n} \rightarrow \infty, B_{n+k} / B_{n} \rightarrow 1 \text { as } n \rightarrow \infty \text { for every fixed integer } k .
$$

(5.5) shows that (1.2) is satisfied for integral $t$ and $a$ with $k(n)=B_{n}$. Making the obvious changes in the argument to suit the discrete case, Theorems 1 and 2 imply 
TheоReм 5. Let (5.2) and (5.3) hold. If the series $\sum_{1}^{\infty} B_{n}^{-1}$ diverges, then the dissipative part of $\Omega$ (which now is the space of infinite sequences of real numbers) has zero m-measure and the coordinate shift transformation $T$ is metrically transitive.

An application of Hopf's theorem then yields (5.1).

It is worth noting that the full force of the assumption of the divergence of $\sum_{1}^{\infty} B_{n}^{-1}$ is used in proving the absence of the dissipative part while the metric transitivity of $T$ needs in its proof merely the fact that $B_{n}$ satisfies (5.5). On the other hand if $\sum_{1}^{\infty} B_{n}^{-1}$ converges, there can be no interval recurrence and hence (5.1) cannot be true. The proof of this observation made in [5] involves an application of the criterion given in [6]. Combining these remarks with Theorem 5 we have the following

CoROllary 1. If the y's are random variables satisfying (5.2) and (5.3), then (5.1) is true or not according as

$$
\sum_{1}^{\infty} B_{n}^{-1}=\infty \quad \text { or } \quad \sum_{1}^{\infty} B_{n}^{-1}<\infty .
$$

An interesting property of the norming constants $B_{n}$ when $\alpha>1$ emerges from the above corollary if we assume Theorem 4 of [2].

Corollary 2. Let (5.2) hold and let the y's belong to the domain of attraction of the symmetric stable law with $\alpha>1$ (with the assumption of finite second moment if $\alpha=2$ ). Then $\sum_{1}^{\infty} B_{n}^{-1}=\infty$.

Proof. We have

$$
\lim _{n \rightarrow \infty} \operatorname{Prob}\left[\frac{y_{1}+\cdots+y_{n}}{B_{n}}-A_{n} \leqq y\right]=V_{\alpha}(y),
$$

where the $A_{n}$ 's are real constants. Since $\alpha>1, E y$ is finite and by a result due to Gnedenko and Korolyuk [8],

$$
A_{n}=\left(n / B_{n}\right) E y \text {. }
$$

Writing $x_{j}=y_{j}-E y_{j}$, since $E x_{j}=0$ and $F^{\prime}(y)$ exists, Theorem 4 of [2] implies (5.1) for every $a$. Corollary 2 then follows from Corollary 1.

We suppose next that the $y$ 's are integer-valued random variables. Then if $\xi$ is an integer

$$
P(n, \xi ; A)=\int_{A} d F_{n}(y-\xi)=\int_{A} p_{n}(k-\xi) \Pi(d k),
$$

where 


$$
\Pi(A)=\text { number of integers in } A,
$$

and for every integer $k$

$$
p_{n}(k)=\operatorname{Prob}\left(y_{1}+\cdots+y_{n}=k\right) .
$$

It is easy to see that

$$
\int_{-\infty}^{\infty} P(n, \xi ; A) \Pi(d \xi)=\Pi(A) .
$$

THEOREM 6. Let the integer-valued random variables $y_{j}$ satisfy the following conditions:

(5.11) The greatest common divisor of the differences of the values which $y_{j}$ assumes with positive probability is one. (This is the condition $(\omega)$ of [7]. It ensures the fact that the span of the lattice distribution $F(y)$ is maximal.)

$$
\lim _{n \rightarrow \infty} \operatorname{Prob}\left[\frac{y_{1}+\cdots+y_{n}}{B_{n}} \leqq y\right]=V_{\alpha}(y) \quad(0<\alpha \leqq 2)
$$

(if $\alpha=2$ we again assume $\left.E y^{2}<\infty\right), V_{\alpha}(y)$ being as in (5.3). Then, provided

$$
\sum_{1}^{\infty} B_{n}^{-1}=\infty
$$

we have

$$
\operatorname{Prob}\left[\lim _{n \rightarrow \infty} \frac{\sum_{1}^{n} f\left(y_{1}+\cdots+y_{i}\right)}{\sum_{1}^{n} g\left(y_{1}+\cdots+y_{i}\right)}=\frac{\sum_{-\infty}^{\infty} f(k)}{\sum_{-\infty}^{\infty} g(k)}\right]=1
$$

for every two functions of integers $f(k), g(k)$ such that

$$
\sum_{-\infty}^{\infty}|f(k)|<\infty, \quad \sum_{-\infty}^{\infty}|g(k)|<\infty, \quad \sum_{-\infty}^{\infty} g(k) \neq 0 .
$$

Proof. An argument of Gnedenko and Kolmogorov [7, pp. 252253] leads to

$$
B_{n} p_{n}(k)=V_{\alpha}^{\prime}\left(k / B_{n}\right)+R_{n},
$$

where $R_{n}$ tends to zero uniformly in $k$. Since the density $V_{\alpha}^{\prime}(y)$ is bounded and continuous and since $V_{\alpha}^{\prime}(0) \neq 0$, we have

$$
B_{n} p_{n}(k) \leqq C \quad \text { independent of } n \text { and } k \text {, }
$$


and

$$
\lim _{n \rightarrow \infty} B_{n} p_{n}(k)=V_{\alpha}^{\prime}(0) \text {. }
$$

The rest of the proof differs from that of Theorem 5 in minor details. The analogues of Theorems 1 and 2 are established by making the necessary changes using II-measure instead of Lebesgue measure. We omit the proof of the following

COROLlaRY. Let the integer-valued random variables $y_{j}$ belong to the domain of attraction of a symmetric stable law with $\alpha>1$. Then (5.14) is true provided $\sum_{1}^{\infty} B_{n}^{-1}$ diverges.

\section{REFERENCES}

1. G. Kallianpur and H. Robbins, Ergodic property of the Brownian motion process, Proc. Nat. Acad. Sci. U.S.A. vol. 39 (1953).

2. T. E. Harris and H. Robbins, Ergodic theory of Markov chains admitting an infinite invariant measure, Proc. Nat. Acad. Sci. U.S.A. vol. 39 (1953).

3. J. L. Doob, Stochastic processes, New York, Wiley, 1953.

4. E. Hopf, Ergodentheorie, New York, Chelsea, 1948.

5. G. Kallianpur and H. Robbins, The sequence of sums of independent random variables, Duke Math. J. vol. 21 (1954) pp. 285-307.

6. K. L. Chung and W. Fuchs, On the distribution of values of sums of random variables, Memoirs of the American Mathematical Society, vol. 6, 1951.

7. B. V. Gnedenko and A. N. Kolmogorov, Predelniie raspredelenia dlia soum nezavisimikh sluchainikh velichin, Moscow-Leningrad, 1949.

8. B. V. Gnedenko and V. S. Korolyuk, Some remarks on the theory of domains of attraction of stable distributions, Dopovidi Akad. Nauk, Ukrain. R.S.R. (1950) (Ukrainian with Russian summary).

Indian Statistical Institute 